\title{
DIFFERENTIAL PROPERTIES OF SOME DENSE SUBALGEBRAS OF $C^{*}$-ALGEBRAS
}

\author{
by E. KISSIN and V. S. SHULMAN*
}

(Received 20th September 1992)

\begin{abstract}
The paper studies some classes of dense *-subalgebras $B$ of $C^{*}$-algebras $A$ whose properties are close to the properties of the algebras of differentiable functions. In terms of a set of norms $\left\{\|\|_{i}\right\}_{i=1}^{P}$ on $B$ it defines $\left(D_{p}^{*}\right)$-subalgebras of $A$ and establishes that they are locally normal $Q^{*}$-subalgebras. If $x=x^{*} \in B$ and $f(t)$ is a function on $S p(x)$, some sufficient conditions are given for $f(x)$ to belong to $B$. For $p=1$, in particular, it is shown that $\left(D_{1}^{*}\right)$-subalgebras are closed under $C^{\infty}$-calculus. If $\delta$ is a closed derivation of $A$, the algebras $D\left(\delta^{p}\right)$ are $\left(D_{p}^{*}\right)$-subalgebras of $A$. In the case when $\delta$ is a generator of a one-parameter semigroup of automorphisms of $A$, it is proved that, in fact, $D\left(\delta^{p}\right)$ are $\left(D_{i}^{*}\right)$-subalgebras. The paper also characterizes those Banach *-algebras which are isomorphic to $\left(D_{1}^{*}\right)$-subalgebras of $C^{*}$-algebras.
\end{abstract}

1991 Mathematics subject classification: 46H20, 46L57.

\section{Introduction}

It is well-known that $C^{*}$-algebras are noncommutative analogies of the algebras of continuous functions. This paper studies some classes of dense *-subalgebras of $C^{*}$ algebras whose properties are "close" to the properties of the algebras of differentiable functions.

In [8] the authors investigated dense locally normal $Q^{*}$-subalgebras $B$ of $C^{*}$-algebras $A$. These subalgebras retain many properties of the enveloping $C^{*}$ algebras: $S p_{A}(x)=$ $S p_{B}(x), x \in B$, every finite-dimensional semisimple representation of $B$ is automatically continuous and extends to $A$ and, for every injective *-homomorphism $\varphi$ of $B$ into a Banach *-algebra, $\|x\| \leqq\|\varphi(x)\|, x \in B$. All closed (in the topology of $A$ ) two-sided ideals of $B$ are obtained by the mapping $I \rightarrow I \cap B$ of the set of all closed two-sided ideals $l$ in $A$, this mapping is one-to-one and it maps the set of all maximal ideals in $A$ onto the set of all maximal ideals in $B$. From Longo's result [9] it also follows that everywhere defined derivations from $B$ into $A$ are automatically bounded.

Let $B$ be a dense *-subalgebra of a $C^{*}$-algebra $\left(A,\|\|_{0}\right)$ and a Banach *-algebra with respect to a norm \|\| . The main motivation of this paper is to describe in terms of the norm \|\| different classes of locally normal $Q^{*}$-subalgebras of $A$. If, for example, \|\| is such that $B$ is closed under $C^{\infty}$-functional calculus of selfadjoint elements, then $B$ is a locally normal $Q^{*}$ subalgebra of $A$. Thus if $\delta$ is a closed *-derivation of $A$, if

\footnotetext{
*The authors are grateful to the University of North London for inviting Victor Shulman to Britain for a three months research visit.
} 
$x=x^{*} \in D\left(\delta^{p}\right)$ and a function $f(t)$ has $p+1$ continuous derivatives, it follows from the result of Bratteli, Elliott and Jorgensen [3] that $f(x) \in D\left(\delta^{p}\right)$, so that $D\left(\delta^{p}\right)$ is a locally normal $Q^{*}$-subalgebra of $A$.

Blackadar and Cuntz [1] started the study of smooth *-subalgebras of $C^{*}$-algebras. The basic concept in their approach is the one of differential seminorms which generalize the seminorms associated with the powers of derivations. They showed that any subalgebra which is complete with respect to a differential seminorm of total order $k$ is closed under $C^{k+1}$-functional calculus.

In this paper we consider $\left(D_{p}\right)$-subalgebras $B$ of Banach algebras $\left(A,\|\|_{0}\right)$ : dense subalgebras of $A$ for which there exist norms $\left\{\|\|_{i}\right\}_{i=0}^{p}$ and positive constants $\left\{D_{i}\right\}_{i=1}^{p}$ such that $\left(B,\|\|_{p}\right)$ is a Banach algebra and

$$
\|x y\|_{i} \leqq D_{i}\left(\|x\|_{i}\|y\|_{i-1}+\|x\|_{i-1}\|y\|_{i}\right), \quad x, y \in B \text { and } 1 \leqq i \leqq p .
$$

The differential subalgebras of order $p$ studied by Blackadar and Cuntz are $\left(D_{p}\right)$-subalgebras and, for $p=1$, these classes coincide. But for $p \geqq 2$, the growth of \|\|$_{p}$ on products and exponentials in $\left(D_{p}\right)$-subalgebras, which determines the properties of the subalgebra, is much faster than in the differential subalgebras. Because of this, even for $p=2$, it is not clear whether $\left(D_{p}\right)$-subalgebras are closed under $C^{\infty}$-functional calculus.

In Theorem 5 we show that if $A$ contains an identity 1 , then $1 \in B$ and $B$ is a $Q$ subalgebra of $A$, i.e., $S p_{B}(x)=S p_{A}(x)$, for all $x \in B$. For the case when $A$ is a $C^{*}$-algebra, $x=x^{*} \in B$ and $f(t)$ is a function on $S p(x)$, Theorem 12 gives some sufficient conditions for $f(x)$ to belong to $B$. Although this condition is much stronger than the condition of Bratteli, Elliott and Jorgensen [3] for the algebras $D\left(\delta^{p}\right)$ and than the condition of Blackadar and Cuntz [1] for differential algebras, nevertheless, as a corollary of this result, we obtain that $\left(D_{p}\right)$-subalgebras of $C^{*}$-algebras are locally normal $Q^{*}$-subalgebras (similarly, the Fourier-Wiener algebra is a locally normal $Q^{*}$-subalgebra, but it is not closed under $C^{\infty}$-functional calculus).

Blackadar and Cuntz [1] studied a special class of flat differential seminorms. They showed that a differential seminorm $T=\left\{\|_{i}\right\}_{i=0}^{p}$ on $B$ is flat if and only if there exist a seminormed algebra $D$ and a derivation $\delta$ of $D$ such that $B \subseteq D\left(\delta^{p}\right)$ and that $|x|_{i}=\left\|\delta^{i}(x)\right\|_{D} / i !, x \in B$ and $0 \leqq i \leqq p$. In Section 3 we show that if $\delta$ is a generator of a one-parameter semigroup of automorphisms of $A$, then the flat differential seminorm $T=\left\{\left\|\delta^{i}(x)\right\|_{0} / i !\right\}_{i=0}^{p}$ of order $p>1$ on $D\left(\delta^{p}\right)$ is equivalent to the differential seminorm $T^{\prime}=\left\{\|\|_{0},\|\|_{p}\right\}$ of order 1, where $\|x\|_{p}=\sum_{i=0}^{p}\left\|\delta^{i}(x)\right\|_{0} / i !$. Thus, in this case, the algebras $D\left(\delta^{p}\right)$ are, in fact, $\left(D_{1}\right)$-subalgebras of $A$.

$\left(D_{1}\right)$-subalgebras of $C^{*}$-algebras constitute probably the most interesting subclass of subalgebras of $C^{*}$-algebras. Section 5 characterizes those Banach *-algebras which are isomorphic to $\left(D_{1}\right)$-subalgebras of $C^{*}$-algebras.

The first version of the article was written before we heard about the paper by Blackadar an Cuntz; several changes were made after we received a preprint of their paper kindly sent to us by Professor Cuntz. We would also like to thank Professor V. I. Burenkov for useful information about the norms of the derivations of functions used in Theorem 8. 


\section{2. (Dp)-Subalgebras of Banach algebras}

Let

$$
a(k, j)=\left(\begin{array}{l}
k \\
j
\end{array}\right)=\left\{\begin{array}{lll}
k ! / j !(k-j) !, & \text { if } j \leqq k \\
0, & \text { if } j>k
\end{array}\right.
$$

By induction one can prove the following formula:

$$
\sum_{k=0}^{m}\left(\begin{array}{l}
k \\
j
\end{array}\right)=\sum_{k=j}^{m}\left(\begin{array}{l}
k \\
j
\end{array}\right)=\left(\begin{array}{c}
m+1 \\
j+1
\end{array}\right), \quad j \leqq m .
$$

Set

$$
S(k, p)=\sum_{j=0}^{p-1}\left(\begin{array}{l}
k \\
j
\end{array}\right)
$$

Making use of (1), we obtain that

$$
\begin{aligned}
& \sum_{k=0}^{m} S(k, p)=\sum_{k=0}^{m} \sum_{j=0}^{p-1}\left(\begin{array}{c}
k \\
j
\end{array}\right)=\sum_{j=0}^{p-1} \sum_{k=0}^{m}\left(\begin{array}{l}
k \\
j
\end{array}\right) \\
& =\sum_{j=0}^{p-1}\left(\begin{array}{c}
m+1 \\
j+1
\end{array}\right)=\sum_{j=1}^{p}\left(\begin{array}{c}
m+1 \\
j
\end{array}\right)=S(m+1, p+1)-1 .
\end{aligned}
$$

Lemma 1. Let $B$ be an algebra and let $\left\{f_{i}\right\}_{i=0}^{p}$ be a set of non-negative functions on $B$ such that for all $i=0, \ldots, p, f_{i}\left(x^{n+m}\right) \leqq f_{i}\left(x^{n}\right) f_{i}\left(x^{m}\right), x \in B$ and $m, n>0$. If there exist positive numbers $\left.\left\{C_{i}\right\}\right\}_{l=1}$ such that

$$
f_{i}\left(x^{2}\right) \leqq C_{i} f_{i-1}(x) f_{i}(x), x \in B \text { and } i=1, \ldots, p,
$$

then, for all $k$,

$$
f_{i}\left(x^{2^{k}}\right) \leqq f_{0}(x)^{2^{k}-S(k, i)} \prod_{j=0}^{i-1}\left[f_{i-j}(x)\right]^{a(k, j)}\left(C_{i-j}\right)^{a(k, j+1)}
$$

In particular, $f_{1}\left(x^{2^{k}}\right) \leqq C_{1}^{k}\left[f_{0}(x)\right]^{2^{k}-1} f_{1}(x)$.

Proof. We have that

$$
f_{i}\left(x^{2^{k}}\right) \leqq C_{i} f_{i-1}\left(x^{2^{k-1}}\right) f_{i}\left(x^{2^{k-1}}\right) \leqq \cdots \leqq C_{i}^{k} f_{i-1}\left(x^{2^{k-1}}\right) f_{i-1}\left(x^{2^{k-2}}\right) \ldots f_{i-1}\left(x^{2}\right) f_{i-1}(x) f_{i}(x)
$$

Since $f_{0}\left(x^{m}\right) \leqq f_{0}(x)^{m}$, we obtain that $f_{1}\left(x^{2^{k}}\right) \leqq C_{1}^{k}\left[f_{0}(x)\right]^{2^{k}-1} f_{1}(x)$ and the lemma holds for $i=1$. Suppose that the lemma holds for $i \leqq n$. Let $i=n+1$. By (3), 


$$
\begin{aligned}
& f_{n+1}\left(x^{2^{k}}\right) \leqq C_{n+1}^{k}(x) f_{n+1}(x) \prod_{m=0}^{k-1} f_{n}\left(x^{2 m}\right) \\
& \left.\leqq C_{n+1}^{k} f_{n+1}(x) \prod_{m=0}^{k-1} f_{0}(x)^{2 m-S(m, n)}\right) \prod_{j=0}^{n-1}\left[f_{n-j}(x)\right]^{a(m, j)}\left(C_{n-j}\right)^{a(m, j+1)}
\end{aligned}
$$

By (2), $\sum_{m=0}^{k-1}\left(2^{m}-S(m, n)\right)=2^{k}-1-S(k, n+1)+1=2^{k}-S(k, n+1)$. By (1), $\sum_{m=0}^{k-1} a(m, j)=$ $a(k, j+1)$. Therefore

$$
\begin{aligned}
& f_{n+1}\left(x^{2^{k}}\right) \leqq C_{n+1}^{k} f_{n+1}(x) f_{0}(x)^{2^{k}-S(k, n+1)} \prod_{j=0}^{n-1} f_{n-j}(x)^{a(k, j+1)} C_{n-j}^{a(k, j+2)} \\
& =f_{0}(x)^{2^{k}-S(k, n+1)} \prod_{j=0}^{n} f_{n+1-j}(x)^{a(k, j)} C_{n+1-j}^{a(k, j+1)} .
\end{aligned}
$$

Corollary 2. Let $2^{m} \leqq n<2^{m+1}$ and set $K(x)=\max _{1 \leqq i \leqq p}\left\{C_{i}, f_{i}(x), 1\right\}$. Then for $m>2 p$,

$$
S(m, p)<m^{p-1} \text { and } f_{p}\left(x^{n}\right) \leqq K(x)^{2(m+1)^{p+1}}\left[f_{0}(x)\right]^{n-d(n)}
$$

where $S(m, p) \leqq d(n) \leqq S(m+1, p+1)$.

Proof. By Lemma 1, $f_{p}\left(x^{2^{i}}\right) \leqq K(x)^{b_{i}}\left[f_{0}(x)\right]^{2^{i}-S(i, p)}$, where

$$
b_{i}=\sum_{j=0}^{p-1}\left(\left(\begin{array}{c}
i \\
j
\end{array}\right)+\left(\begin{array}{c}
i \\
j+1
\end{array}\right)\right)=2 S(i, p)-1+\left(\begin{array}{l}
i \\
p
\end{array}\right) \leqq 2 S(i, p+1)
$$

Set $f_{p}\left(x^{0}\right)=1$. Let $n=\sum_{i=0}^{m} a_{i} 2^{i}$, where $a_{i}$ are either 1 or 0 and $a_{m}=1$. Then

$$
\begin{aligned}
& f_{p}\left(x^{n}\right) \leqq \prod_{i=0}^{m} f_{p}\left(x^{a_{i} 2^{i}}\right) \leqq \prod_{i=0}^{m} K(x)^{a_{i} b_{i}}\left[f_{0}(x)\right]^{a_{i}\left(2^{i}-S(i, p)\right)} \\
& \leqq K(x)^{b}\left[f_{0}(x)\right]^{n-d(n)}
\end{aligned}
$$

where $b=\sum_{i=0}^{m} a_{i} b_{i}$ and $d(n)=\sum_{i=0}^{m} a_{i} S(i, p)$. Since $a_{m}=1$, we obtain from (2) that $b \leqq 2 S(m+1, p+2)$ and $S(m, p) \leqq d(n) \leqq S(m+1, p+1)$.

For $m>2 p$, we have that $S(m, p)<p\left(\begin{array}{c}m \\ p-1\end{array}\right)<m^{p-1}$. Since $1 \leqq K(x)$, then $K(x)^{b} \leqq$ $K(x)^{2(m+1)^{p+1}}$.

Definition. Let $\left\{\|\|_{i}\right\}_{i=0}^{P}$ be algebraic seminorms on an algebra $B$, i.e., $\|x y\|_{i} \leqq\|x\|_{i}\|y\|_{i}$.

(i) We say that $B$ has property $\left(\mathbf{D}_{p}\right)$ with respect to $\left\{\|\|_{i}\right\}_{i=0}$ if there exist numbers $\left\{D_{i}\right\}_{i=1}^{p}, D_{i} \geqq 0$, such that for all $x \in B$, 


$$
\|x y\|_{i} \leqq D_{i}\left(\|x\|_{i}\|y\|_{i-1}+\|x\|_{i-1}\|y\|_{i}\right), \quad 1 \leqq i \leqq p .
$$

(ii) Let \|\|$_{0}$ and \|\|$_{p}$ be norms on $B$ and let $A$ be the completion of $B$ with respect to \|\|$_{0}$. If $B$ is a Banach algebra with respect to \|\|$_{p}$, then we say that $B$ is a $\left(\mathrm{D}_{p}\right)$-subalgebra of $A$. If, in addition, $B$ is a ${ }^{*}$-algebra and $\left\|x^{*}\right\|_{i}=\|x\|_{i}, 0 \leqq i \leqq p$, then we say that $B$ is a $\left(D_{p}^{*}\right)$-subalgebra of the Banach *-algebra $A$.

Remark. Let $B$ have property $\left(D_{p}\right)$. Set $R=\max _{1 \leq i \leq p}\left(1, D_{i}\right)$ and $|x|_{i}=R\|x\|_{i}$. Then $R \geqq 1$, all $\mid \|_{i}$ are algebraic seminorms on $B$ equivalent to \|\|$_{i}$ and $|x y|_{i} \leqq|x|_{i}|y|_{i-1}+|x|_{i-1}|y|_{i}$. Hence $B$ has property $\left(D_{p}\right)$ with respect to $\left\{\|_{i}\right\}_{i=0}^{p}$.

Let $B$ be a $\left(\mathbf{D}_{p}\right)$-subalgebra of $A$ and $E$ be a $\left(\mathbf{D}_{q}\right)$-subalgebra of $B$. If $E$ is dense in $A$, then $E$ is a $\left(D_{p+q}\right)$-subalgebra of $A$. In particular, if the norm \|\|$_{0}$ is continuous on $B$ with respect to the norm \|\|$_{p}$, then $E$ is dense in $A$ with respect to \|\|$_{0}$.

Since $\left\|x^{n+m}\right\| \leqq\left\|x^{n}\right\|\left\|x^{m}\right\|$ for any seminorm \|\| , Lemma 1 and Corollary 2 hold if $B$ has property $\left(\mathrm{D}_{p}\right)$ with $C_{i}=2 D_{i}$.

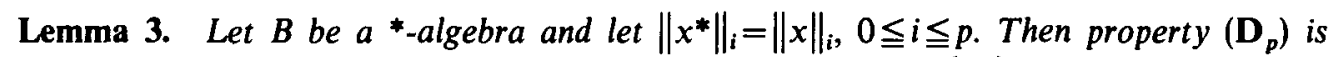
equivalent to the following property $\left(D_{p}^{*}\right)$ : there exist numbers $\left.\left\{D_{i}^{\prime}\right\}\right\}_{i=1}, D_{i}^{\prime} \geqq 0$, such that for all $x \in B$,

$$
\left\|x^{*} x\right\|_{i} \leqq D_{i}^{\prime}\|x\|_{i}\|x\|_{i-1}, \quad 1 \leqq i \leqq p
$$

Proof. Let $x=a+i b \in B$. Then $\|a\|_{i}=\left\|\left(x+x^{*}\right) / 2\right\|_{i} \leqq\|x\|_{i}$ and $\|b\|=\left\|\left(x-x^{*}\right) / 2 i\right\| \leqq\|x\|_{i}$. Property $\left(\mathbf{D}_{p}\right)$ clearly implies property $\left(\mathbf{D}_{p}^{*}\right)$. Let $B$ have property $\left(\mathbf{D}_{p}^{*}\right)$. Fix $i$ and set \|\|$=\|\|_{i},\|=\| \|_{i-1}$ and $D=D_{i}^{\prime}$. We have that

$$
x^{2}=a^{2}+i(a b+b a)-b^{2}=(1-i) a^{2}-(1+i) b^{2}+i(a+b)^{2}, \quad x^{*} x=a^{2}+i(a b-b a)+b^{2} .
$$

Then

$$
\|a b-b a\|=\left\|x^{*} x-a^{2}-b^{2}\right\| \leqq D(\|x\||x|+\|a\||a|+\|b\||b|) \leqq 3 D\|x\||x| .
$$

For $t>0$,

$$
\begin{aligned}
& \|2 t(a b+b a)\|=\left\|(a+t b)^{2}-(a-t b)^{2}\right\| \leqq\left\|(a+t b)^{2}\right\|+\left\|(a-t b)^{2}\right\| \\
& \quad \leqq D(\|a+t b\||a+t b|+\|a-t b\||a-t b|) \leqq 2 D\left(\|a\||a|+t|a|\|b\|+t\|a\||b|+t^{2}\|b\||b|\right) .
\end{aligned}
$$

Therefore

$$
\|a b+b a\| \leqq D(\|a\||b|+|a|\|b\|+t\|b\||b|+\|a\||a| / t) .
$$

Using the inequality $2(\lambda \mu)^{1 / 2} \leqq \lambda+\mu$, we obtain that

$$
\min (t\|b\||b|+\|a\||| a \mid / t)=2(\|b\||b|\|a\||a|)^{1 / 2} \leqq\|a\||| b|+| a \mid\|b\| .
$$


Therefore $\|a b+b a\| \leqq 2 D(\|a\||b|+|a|\|b\|)$, so that $\|a b+b a\| \leqq 4 D\|x\||x|$. Hence we obtain that $\|a b\|=\|(a b+b a)+(a b-b a)\| / 2 \leqq 3.5 D\|x\||x|$. Since $\|x\| \leqq\|a\|+\|b\|$ and $|x| \leqq|a|+|b|$,

$$
\|a b\| \leqq 3.5 D(\|a\|+\|b\|)(|a|+|b|) \text {. }
$$

Let now $x=a_{1}+i a_{2}$ and $y=b_{1}+i b_{2}$. For every $j$ and $k$,

$$
\left\|a_{j} b_{k}\right\| \leqq 3.5 D\left(\left\|a_{j}\right\|+\left\|b_{k}\right\|\right)\left(\left|a_{j}\right|+\left|b_{k}\right|\right) \leqq 3.5 D(\|x\|+\|y\|)(|x|+|y|) .
$$

Then

$$
\|x y\| \leqq \sum_{j, k=1}^{2}\left\|a_{j} b_{k}\right\| \leqq 14 D(\|x\|+\|y\|)(|x|+|y|)
$$

Set $t=|x|, s=|y|, u=x / t$ and $v=y / s$. Then $|u|=1,|v|=1$ and

$$
\|x y\|=t s\|u v\| \leqq 28 t s D(\|u\|+\|v\|)=28 D(\|x\||y|+|x|\|y\|) .
$$

Thus $B$ has property $\left(\mathbf{D}_{p}\right)$.

Recall that a normed algebra $B$ with identity is a $Q$-algebra if the group of all invertible elements in $B$ is open in $B$. If, in addition, $B$ is a ${ }^{*}$-normed algebra, then $B$ is a $Q^{*}$-algebra. Let $S p_{B}(x)$ be the spectrum and $r_{B}(x)$ be the spectral radius of $x$ in $B$.

Lemma 4. $([8,11])$. The following conditions are equivalent:

(i) $B$ is a Q-algebra;

(ii) $r_{B}(x) \leqq\|x\|$ for all $x \in B$;

(iii) $S p_{A}(x)=S p_{B}(x)$ for all $x \in B$, where $A$ is the completion of $B$.

Bratteli and Robinson [4] (cf. [5]) proved that if $\delta$ is a closed *-derivation of a $C^{*}$-algebra with an identity 1 , then 1 belongs automatically to the domain $D(\delta)$ of $\delta$. In [8] this result was extended to the case when $\delta$ is a densely defined closed derivation of a Banach algebra $A$. The following theorem shows that $\left(D_{p}\right)$-subalgebras of Banach algebras are $Q$-algebras and that 1 automatically belongs to them.

Theorem 5. Let $B$ be $a\left(\mathbf{D}_{p}\right)$-subalgebra of $A$ and let $A$ contain an identity 1. Then $1 \in B$ and $B$ is a $Q$-algebra with respect to \|\|$_{0}$.

Proof. Let $y$ be an element in $B$ such that $\|1-y\|_{0}=\varepsilon<1$. Set

$$
a_{n}=1-(1-y)^{n}=-\sum_{i=1}^{n}\left(\begin{array}{c}
n \\
i
\end{array}\right)(-y)^{i} \in B .
$$


Then $a_{n+1}-a_{n}=(1-y)^{n} y$. Let $K=K(1-y)=\max _{1 \leqq i \leqq p}\left\{2 D_{i},\|1-y\|_{i}, 1\right\}$. From Corollary 2 we obtain that

$$
\left\|a_{n+1}-a_{n}\right\|_{p} \leqq\left\|(1-y)^{n}\right\|_{p}\|y\|_{p} \leqq K^{2(m+1)^{p+1}} \varepsilon^{n-S(m+1, p+1)}\|y\|_{p} \leqq K^{2(m+1)^{p+1}} \varepsilon^{n-(m+1) p}\|y\|_{p}
$$

where $m \leqq \lg _{2} n \leqq m+1$. The series $\sum_{n=1}^{\infty} K^{2\left(\lg _{2} n+1\right)^{p+1}} \varepsilon^{n-\left(\lg _{2} n+1\right)^{p}}$ converges. Therefore its partial sums $S_{i}$ converge.

For any $q>0$,

$$
\left\|a_{n+q}-a_{n}\right\|_{p} \leqq \sum_{j=n}^{n+q-1}\left\|a_{j+1}-a_{j}\right\|_{p} \leqq\|y\|_{p}\left(S_{n+q-1}-S_{n-1}\right)
$$

Since $B$ is a Banach algebra with respect to \|\|$_{p}$, the sequence $\left\{a_{n}\right\}$ converges to an element $x$ in $B$. Hence $\left\{a_{n} y\right\}$ converges to $x y$ in $B$.

On the other hand,

$$
a_{n} y=y-(1-y)^{n} y=y-\left(a_{n+1}-a_{n}\right)
$$

Since $\left\|a_{n+1}-a_{n}\right\|_{p} \rightarrow 0,\left\{a_{n} y\right\}$ converges to $y$ with respect to \|\|$_{p}$. Thus $x y=y$. Since $\|1-y\|_{0}<1, y$ is invertible in $A$. Therefore $x=1 \in B$.

Since $\left(B,\|\|_{p}\right)$ is a Banach algebra, it follows from Corollary 2 that

$$
r_{B}(x)=\lim _{n \rightarrow \infty}\left(\left\|x^{n}\right\|_{p}\right)^{1 / n} \leqq \lim _{n \rightarrow \infty} K(x)^{2(m+1)^{p+1 / n}}\|x\|_{0}^{(n-d(n)) / n}
$$

where $K(x)=\max _{1 \leqq i \leqq p}\left\{2 D_{i},\|x\|_{i}, 1\right\}$ and where $\left(\begin{array}{c}m \\ p-1\end{array}\right) \leqq d(n) \leqq(m+1)^{p}-1$ and $m \leqq \lg _{2} n<m+1$. Hence $r_{B}(x) \leqq\|x\|_{0}$ and, by Lemma $4, B$ is a $Q$-algebra with respect to \|\|$_{0}$.

Example 1. Let $(A,\|\cdot\|)$ be a Banach algebra. A two-sided ideal $I$ of $A$ is symmetrically normable (see [11]) if $I$ is a Banach algebra with respect to a norm \|\|$_{s}$ and

$$
|x y z|_{s} \leqq\|x\||y|_{s}\|z\|, \quad \text { for } y \in I \text { and } x, z \in A \text {. }
$$

The symmetric Segal algebras of locally compact groups $G$ are symmetrically normable ideals of $L^{1}(G)$ [13]. Shatten classes of operators give another example of symmetrically normable ideals [6].

If $I$ is a dense symmetrically normable ideal in $A$, then it is a $\left(D_{1}\right)$-subalgebra of $A$ with respect to \|\| and $\|_{s}$. By Theorem $5, A$ does not have an identity. The algebras $A$ and $I$ can be canonically embedded in larger Banach algebras $\hat{A}=A+\mathbb{C} 1$ and $\hat{I}=I+\mathbb{C} 1$ with the norms 


$$
\|t \mathbf{1}+x\|=|t|+\|x\| \text { and }\|t 1+y\|_{1}=|t|+|y|_{s}, \quad t \in \mathbb{C}, x \in A, y \in I
$$

respectively. The algebra $\hat{I}$ is a $\left(\mathbf{D}_{1}\right)$-subalgebra of $\hat{A}$.

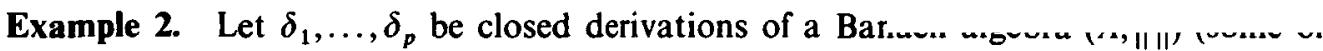
them may be the same). For every subset $S=\left\{k_{1}, \ldots, k_{m}\right\}$ of $\{p, \ldots, 1\}, k_{1}>k_{2} \ldots>k_{m}$, set

$$
\delta_{S}(x)=\delta_{k_{1}}\left(\ldots\left(\delta_{k_{m}}(x)\right) \ldots\right) \text { and } \delta_{\varnothing}(x)=x
$$

where $\varnothing$ is the empty set. Let $D\left(\delta_{S}\right)$ be the domain of $\delta_{S}$ and let $D\left(\delta_{p} \ldots \delta_{1}\right)=\bigcap_{S} D\left(\delta_{S}\right)$ where $S$ ranges over all subsets of $\{p, \ldots, 1\}$.

For $x \in D\left(\delta_{p} \ldots \delta_{1}\right)$, set $\|x\|_{0}=\|x\|$ and, for $1 \leqq i \leqq p$, set

$$
\|x\|_{i}=\sum_{S}\left\|\delta_{S}(x)\right\|
$$

where $S$ ranges over all subsets of $\{i, \ldots, 1\}$. For example,

$$
\|x\|_{1}=\|x\|+\left\|\delta_{1}(x)\right\| \text { and }\|x\|_{2}=\|x\|+\left\|\delta_{1}(x)\right\|+\left\|\delta_{2}(x)\right\|+\left\|\delta_{2}\left(\delta_{1}(x)\right)\right\| .
$$

For every subset $S$ of $\{p, \ldots, 1\}$, the derivation property implies the identity $\delta_{S}(x y)=$ $\sum_{Q} \delta_{Q}(x) \delta_{S \backslash Q}(y)$, where $Q$ ranges over all subsets of $S$. Therefore

$$
\|x y\|_{i} \leqq \sum_{S} \sum_{Q}\left\|\delta_{Q}(x)\right\|\left\|\delta_{S \backslash Q}(y)\right\|
$$

where $S$ ranges over all subsets of $\{i, \ldots, 1\}$ and $Q$ ranges over all subsets of $S$. From this we can deduce that

$$
\|x y\|_{i} \leqq\|x\|_{i}\|y\|_{i} \text { and }\|x y\|_{i} \leqq\|x\|_{i}\|y\|_{i-1}+\|x\|_{i-1}\|y\|_{i} .
$$

Therefore $D\left(\delta_{p} \ldots \delta_{1}\right)$ has property $\left(\mathbf{D}_{p}\right)$ with respect to the norms $\left\{\|\|_{i}\right\}_{i=0}$. The closedness of all the derivations $\delta_{1}, \ldots, \delta_{p}$ implies that $D\left(\delta_{p} \ldots \delta_{1}\right)$ is a Banach algebra with respect to \|\|$_{p}$. If $D\left(\delta_{p} \ldots \delta_{1}\right)$ is dense in $A$, then it is a $\left(\mathbf{D}_{p}\right)$-subalgebra of $A$.

Example 3. Let $(A,\|\|)$ be a Banach algebra, let $F$ be a linear closed mapping from a dense subalgebra $D(F)$ of $A$ into a normed space $\left(H,\|\|_{H}\right)$ and let there exist $D \geqq 0$ such that

$$
\|F(a b)\|_{H} \leqq D\left(\|F(a)\|_{H}\|b\|+\|a\|\|F(b)\|_{H}\right), \quad a, b \in D(F) .
$$

For example, $H$ is a Banach $A$-bimodule and $F$ is a closed derivation from $A$ into $H$. Set $\|a\|_{0}=\|a\|$ and $\|a\|_{1}=\|a\|+\|F(a)\|_{H}$. Then $D(F)$ is a $\left(\mathbf{D}_{1}\right)$-subalgebra of $A$ with respect to the norms \|\|$_{0}$ and \|\|$_{1}$. 
Example 4. Let $(\mathscr{A}, \mid)$ and $\left(\mathscr{B}, \|_{1}\right)$ be Banach algebras and let $\mathscr{B}$ be a $\left(D_{1}\right)$-subalgebra of $\mathscr{A}$ with respect to $\|$ and $\|_{1}$. By $C(\mathscr{A})$ and $C(\mathscr{B})$ we denote the Banach algebras of all converging sequences $a=\left\{a_{n}\right\}, a_{n} \in \mathscr{A}$, and $b=\left\{b_{n}\right\}, b_{n} \in \mathscr{B}$ with the norms $\|a\|_{0}=\sup \left|a_{n}\right|$ and $\|b\|_{1}=\sup \left|b_{n}\right|_{1}$ respectively. Then $C(\mathscr{B})$ is a $\left(\mathrm{D}_{1}\right)$-subalgebra of $C(\mathscr{A})$. Let $H=L_{1}(\mathscr{B})$ be the linear manifold in $C(\mathscr{B})$ which consists of all $b=\left\{b_{n}\right\}$ such that

$$
\|b\|_{H}=\sum_{n=1}^{\infty}\left|b_{n}\right|_{1}<\infty
$$

Then $L_{1}(\mathscr{B})$ is a Banach $C(\mathscr{B})$-bimodule. Let

$$
F(b)=\left\{b_{1}, b_{2}-b_{1}, \ldots, b_{n}-b_{n-1}, \ldots\right)
$$

be a mapping from $C(\mathscr{B})$ into $L_{1}(\mathscr{B})$. Then $L_{1}(\mathscr{B}) \subseteq D(F)$ and $D(F)$ contains every constant sequence $b=\left\{b_{n}\right\} \in \mathscr{B}, b_{n}=b_{m}$, for all $n$ and $m$. Hence $D(F)$ is dense in $C(\mathscr{B})$, so that $F$ is a closed derivation from $C(\mathscr{B})$ into $L_{1}(\mathscr{B})$. Thus $D(F)$ is a $\left(D_{1}\right)$-subalgebra of $C(\mathscr{B})$ with respect to the norms $\|b\|_{1}$ and $\|b\|_{2}=\|b\|_{1}+\|F(b)\|_{H}$. It is easy to check that $D(F)$ is also dense in $C(\mathscr{A})$, so that $D(F)$ is a $\left(\mathbf{D}_{2}\right)$-subalgebra of $C(\mathscr{A})$.

\section{Differential subalgebras of Banach algebras}

In this section we consider a special subclass of $\left(\mathbf{D}_{p}\right)$-subalgebras of Banach algebras-differential subalgebras studied by Blackadar and Cuntz [1]. A set of seminorms $T=\left\{|\cdot|_{i}\right)_{i=0}^{p}$ (not necessarily algebraic on an algebra $B$ is called a differential seminorm of order $p$ if

$$
|x y|_{i} \leqq \sum_{j=0}^{i} K_{j, i-j}|x|_{j}|y|_{i-j}, \quad x, y \in B \quad \text { and } 0 \leqq i \leqq p,
$$

where $K_{j, m}$ are nonnegative constants and $K_{0,0}=1$. Differential seminorms $T=\left\{|\cdot|_{i}\right\}_{i=0}^{p}$ and $T^{\prime}=\left\{|\cdot|_{i}^{\prime}\right)_{i=0}^{m}$ are equivalent if the seminorms $\sum_{i=0}^{p} \mid \|_{i}$ and $\sum_{i=0}^{m} \|_{i}^{\prime}$ are equivalent on $B$. Every differential seminorm $T$ is equivalent to a differential seminorm $T^{\prime}=\left\{||_{i}^{\prime}\right)_{i=0}^{m}$ such that $|x y|_{i}^{\prime} \leqq \sum_{j=0}^{i}|x|_{j}^{\prime}|y|_{i-j}^{i}$.

For $0 \leqq i \leqq p$, set $R_{i}=\max _{j+n \leqq i}\left(K_{j, n}\right)$.

Lemma 6. $\|x\|_{i}=R_{i} \sum_{n=0}^{i}|x|_{n}, 0 \leqq i \leqq p$, are algebraic seminorms on $B$ and $B$ has property $\left(\mathrm{D}_{p}\right)$ with respect to $\left\{\|\cdot\|_{i}\right\}_{i=0}$ and with constants $D_{i}=R_{i} / R_{i-1}$.

Proof. We have

$$
\|x y\|_{i}=R_{i} \sum_{m=0}^{i}|x y|_{m} \leqq R_{i} \sum_{m=0}^{i} \sum_{j=0}^{m} K_{j, m-j}|x|_{j}|y|_{m-j} \leqq R_{i}^{2} \sum_{m=0}^{i} \sum_{j=0}^{m}|x|_{j}|y|_{m-j} \leqq\|x\|_{i}\|y\|_{i},
$$


so that \|\|$_{i}$ are algebraic seminorms on $B$. From (4) it follows that

$$
\begin{aligned}
\|x y\|_{i} & =R_{i}^{2} \sum_{m=0}^{i} \sum_{j=0}^{m}|x|_{j}|y|_{m-j} \leqq R_{i}^{2}\left[\left(\sum_{j=0}^{i}|x|_{j}\right)\left(\sum_{j=0}^{i-1}|y|_{j}\right)+\left(\sum_{j=0}^{i-1}|x|_{j}\right)\left(\sum_{j=0}^{i}|y|_{j}\right)\right] \\
& \leqq D_{i}\left(\|x\|_{i}\|y\|_{i-1}+\|x\|_{i-1}\|y\|_{i}\right) .
\end{aligned}
$$

Definition. Let $T=\left\{|\cdot|_{i}\right\}_{i=0}$ be a differential seminorm on $B$, let \|\|$_{0}=\|\|_{0}$ and \|\|$_{p}$ be norms on $B$ and let $\left(B,\|\|_{p}\right)$ be a Banach algebra. By $A$ we denote the completion of $B$ with respect to \|\|$_{0}$. Then $(B, T)$ is called a differential subalgebra of $A$ of order $p$. If, in addition, $B$ is a *algebra and $\left|x^{*}\right|_{i}=|x|_{i}, 0 \leqq i \leqq p$, then $(B, T)$ is a differential *-subalgebra of the Banach *-algebra $A$.

Lemma 1 establishes the growth of $\left\|x^{2^{k}}\right\|_{p}$, as $k \rightarrow \infty$, in the case when $B$ has property $\left(D_{p}\right)$. Lemma 7 below is similar to Lemma 1 and estimates the growth of $\left\|x^{2^{k}}\right\|_{p}$, as $k \rightarrow \infty$, when $B$ has a differential seminorm $T=\left\{|\cdot|_{i}\right\}_{i=0}^{p}$ (cf. [1]). Set $C_{i}=K_{i, 0}+K_{0, i}$ and $N_{i}=\max C_{j_{1}} C_{j_{2}} \ldots C_{j_{n}}, i=1, \ldots, p$, where maximum is taken over all the sets of integers $\left(j_{1}, \ldots, j_{n}\right)$ such that $j_{1}+\ldots+j_{n}=i$. Changing slightly, if necessary, the constants $C_{i}$, we can assume that $C_{i} \neq N_{j} N_{i-j}$ for $1<i$ and $1 \leqq j<i$. Let $N=\max _{1 \leqq i \leqq p} N_{i}$ be the total order of $T$ (cf. [1]).

Lemma 7. Let $x \in B$ and $|x|_{0}=1$. Then there are constants $K_{i}(x), i=1, \ldots, p$, continuous with respect to \|\|$_{p}$ such that, for all $k$,

$$
\left|x^{2^{k}}\right|_{i} \leqq K_{i}(x) N_{i}^{k} \text { and }\left\|x^{2^{k}}\right\|_{p} \leqq K(x) N^{k}, \text { where } K(x)=R_{p} \sum_{i=1}^{p} K_{i}(x) \text {. }
$$

Proof. Let $\left\{u_{k}\right\}_{k=0}^{\infty}, a,\left\{b_{i}\right\}_{i=1}^{m}$ and $\left\{c_{i}\right\}_{i=1}^{m}$ be positive numbers such that $a \neq b_{i}$, $1 \leqq i \leqq p$, and

$$
u_{k} \leqq a u_{k-1}+\sum_{i=1}^{m} c_{i} b_{i}^{k-1}
$$

Let $d=\max _{1 \leqq i \leqq m}\left(a, b_{i}\right)$ and $\lambda=u_{0}+\sum_{i=1}^{m} c_{i} /\left|b_{i}-a\right|$. Then

$$
u_{k} \leqq a^{k} u_{0}+\sum_{i=1}^{m} c_{i}\left(b_{i}^{k}-a^{k}\right) /\left(b_{i}-a\right) \leqq \lambda d^{k}
$$

Set $L_{j, i-j}=K_{j, i-j}+K_{i-j, j}$. Then, for $1 \leqq i \leqq p$,

$$
\left|x^{2^{k}}\right|_{i} \leqq \sum_{j=0}^{i} K_{j, i-j}\left|x^{2^{k-1}}\right|_{j}\left|x^{2^{k-1}}\right|_{i-j} \leqq C_{i}\left|x^{2^{k-1}}\right|_{i}+\sum_{j=1}^{[i / 2]} L_{j, i-j}\left|x^{2^{k-1}}\right|_{j}\left|x^{2^{k-1}}\right|_{i-j}
$$


For $i=1,\left|x^{2^{k}}\right|_{1} \leqq C_{1}\left|x^{2^{k-1}}\right|_{1}=N_{1}\left|x^{2^{k-1}}\right|_{1}$. By (5), $\left|x^{2^{k}}\right|_{1} \leqq K_{1}(x) N_{1}^{k}$. where $K_{1}(x)=|x|_{1}$ is continuous with respect to \|\|$_{p}$. Assume that the lemma holds for $i \leqq m-1$. Since $N_{i} N_{j} \leqq N_{i+j}$, for $i=m$,

$$
\begin{aligned}
\left|x^{2^{k}}\right|_{m} \leqq C_{m} \mid x^{2^{k-1}} & \left.\right|_{m}+\sum_{j=1}^{[m / 2]} L_{j, m-j}\left|x^{2^{k-1}}\right| j\left|x^{2^{k-1}}\right|_{m-j} \\
& \leqq C_{m}\left|x^{2^{k-1}}\right|_{m}+\sum_{j=1}^{[m / 2]} L_{j, m-j} K_{j}(x)\left(N_{j} N_{m-j}\right)^{k-1}
\end{aligned}
$$

Since $N_{m}=\max _{j}\left(C_{m}, N_{j} N_{m-j}\right)$, it follows from (5) that $\left|x^{2^{k-1}}\right|_{m} \leqq K_{m}(x) N_{m}^{k}$, where $K_{m}(x)$ is continuous with respect to \|\|$_{p}$.

We also have that

$$
\left\|x^{2^{k}}\right\|_{p}=R_{p} \sum_{i=1}^{p}\left|x^{2^{k}}\right|_{i} \leqq R_{p} \sum_{i=1}^{p} K_{i}(x) N_{i}^{k} \leqq K(x) N^{k} .
$$

Let a graded algebra $\mathscr{B}=B_{0}+\ldots+B_{p}$ be the direct sum of subspaces $B_{i}$, let $B_{i} B_{j} \subseteq B_{i+j}$ (we assume that $B_{n}=\{0\}$ if $p<n$ ) and let $P_{i}$ be the projections onto $B_{i}$. Then $B_{0}$ is a subalgebra of $\mathscr{B}$ and all $B_{i}$ are $B_{0}$-bimodules. Suppose that every $B_{i}$ has a space seminorm $s_{i}(x)$ and that there are constants $K_{i, j}$ such that $K_{0,0}=1$, and

$$
s_{i+j}(x y) \leqq K_{i, j} s_{i}(x) s_{j}(y), \quad x \in B_{i} \text { and } y \in B_{j}
$$

For $x \in B$, set $S_{i}(x)=s_{i}\left(P_{i}(x)\right), 0 \leqq i \leqq p$. Then $S_{i}$ are seminorms on $\mathscr{B}, x=\sum_{i=0}^{p} P_{i}(x)$ and

$$
\begin{aligned}
S_{i}(x y) & =s_{i}\left(P_{i}(x y)\right)=s_{i}\left(\sum_{j=0}^{i} P_{j}(x) P_{i-j}(y)\right) \leqq \sum_{j=0}^{i} s_{i}\left(P_{j}(x) P_{i-j}(y)\right) \\
& \leqq \sum_{j=0}^{i} K_{j, i-j} s_{j}\left(P_{j}(x)\right) s_{i-j}\left(P_{i-j}(y)\right)=\sum_{j=0}^{i} K_{j, i-j} S_{j}(x) S_{i-j}(y) .
\end{aligned}
$$

Therefore $S=\left\{S_{i}\right)_{i=0}^{p}$ is a differential seminorm on $\mathscr{B}$. If all $s_{i}$ are norms on $B_{i}$, then, by (4), $\mathscr{B}$ is a graded normed algebra with respect to the norm $\|x\|_{p}=R_{p} \sum_{i=0}^{P} S_{i}(x)$.

As an example, we can consider a nest algebra $\mathscr{B}=\operatorname{Alg} N=\left\{V \in B(H): V L_{k} \subseteq L_{k}\right\}$, where $N$ is a nest of subspaces $\left.\left\{L_{k}\right\}\right\}_{k=0}^{+1},\{0\}=L_{0} \subset L_{1} \subset \ldots \subset L_{p+1}=H$, of a Hilbert space $H$. Let $Q_{k}$ be the projections on $L_{k}$ and let $R_{k}=Q_{k}-Q_{k-1}, k=1, \ldots, p+1$. For $i=0, \ldots, p$, set

$$
B_{i}=\left\{V \in \mathscr{B}: V R_{k}=R_{k-i} V R_{k} \text {, for all } k=1, \ldots, p+1\right\}
$$

and $s_{i}(V)=\|V\|, V \in B_{i}$, where \|\| is the usual norm of the operator $V$ on $H$. Let $P_{i}$ be the projections onto $B_{i}$. Then $\mathscr{B}=B_{0}+\ldots+B_{p}$ is a graded algebra and $S=\left\{S_{i}\right\}_{i=0}^{p}$, where $S_{i}(x)=s_{i}\left(P_{i}(V)\right)$, is a differential seminorm on $\operatorname{Alg} N$ of order $p$. 
A differential seminorm $T=\left\{||_{i}\right\}_{i=0}^{p}$ on an algebra $B$ is called flat ([1, Def. 4.3]) if there is a homomorphism $\phi$ from $B$ into a graded algebra $\mathscr{B}$ with a differential seminorm $S=\left\{S_{i}\right\}_{i=0}^{p}$ such that $|x|_{i}=S_{i}(\phi(x))$.

Let, in particular, all $B_{i}=B_{0}, 1 \leqq i \leqq p$, and $\delta$ be a derivation of $B_{0}$. Set $\varphi_{i}(x)=\delta^{i}(x) / i$ !, $x \in D\left(\delta^{p}\right)$. Since, for every $i$,

$$
\varphi_{i}(x y)=\delta^{i}(x y) / i !=\sum_{j=0}^{i}\left(\begin{array}{l}
i \\
j
\end{array}\right) \delta^{j}(x) \delta^{i-j}(y) / i !=\sum_{j=0}^{i} \varphi_{j}(x) \varphi_{i-j}(y)
$$

the mapping $\phi(x)=\left(x, \varphi_{1}(x), \ldots, \varphi_{p}(x)\right)$ is a homomorphism from $D\left(\delta^{p}\right)$ into $\mathscr{B}$. Let $\|_{0}$ be an algebraic seminorm on $B_{0}$. Set $|x|_{i}=\left|\varphi_{i}(x)\right|_{0}$ for $x \in D\left(\delta^{p}\right)$. Then $T=\left\{|\cdot|{ }_{i}\right)_{i=0}^{p}$ is a flat differential seminorm on $D\left(\delta^{p}\right)$. If $\left(B_{0}, \|_{0}\right)$ is a Banach algebra, $\delta$ is a closed derivation and $D\left(\delta^{p}\right)$ is dense in $B_{0}$, then $D\left(\delta^{p}\right)$ is a differential subalgebra of $B_{0}$ of order $p$.

Blackadar and Cuntz ([1, Th. 4.4]) proved that $T=\left\{\|_{i}\right\}_{i=0}^{p}$ is flat if and only if there exist a seminormed algebra $D$ and a derivation $\delta$ of $D$ such that $B \subseteq D\left(\delta^{p}\right)$ and $|x|_{i}=\left\|\delta^{i}(x)\right\|_{D} / i !, x \in B$ and $0 \leqq i \leqq p$. We shall now show that, under some conditions on $\delta, T$ is equivalent to the differential seminorm $T^{\prime}=\left\{|\cdot|_{0},\|\cdot\|_{p}\right\}$ of order 1 where $\|x\|_{p}=\sum_{i=0}^{p}|x|_{i}$.

Let $\mathscr{T}=\bigcup_{i=1}^{n}\left[a_{i}, b_{i}\right], n<\infty$, be the union of disjoint segments and $C(\mathscr{T})$ be the $C^{*}$-algebra of all continuous functions on $\mathscr{T}$ with the norm $\|f\|=\sup _{t \in \mathscr{T}}|f(t)|$. Let $\delta=d / d t$. For any $p, D\left(\delta^{p}\right)=D_{p}(\mathscr{T})$ is a dense *-subalgebra of $C(\mathscr{T})$ which consists of all functions $f(t)$ such that $f^{(p)}(t) \in C(\mathscr{T})$. Set $|f|_{k}=\left\|f^{(k)}\right\| / k !$. Then $T=\left\{|\cdot|_{k}\right)_{k=0}$ is a differential seminorm on $C(\mathscr{T})$ of order $p$. For $0 \leqq m \leqq p$,

$$
\|f\|_{m}=\sum_{k=0}^{m}\left\|f^{(k)}\right\| / k !
$$

are norms on $D_{p}(\mathscr{T})$ and $D_{p}(\mathscr{T})$ is a Banach *-algebra with respect to \|\|$_{p}$.

Theorem 8. $T^{\prime}=\left\{\|\cdot\|_{0},\|\cdot\|_{p}\right\}$ is a differential seminorm of order 1 on $D_{p}(\mathscr{T})$ equivalent to $T$, i.e., there exists $D_{1}(p)>0$ such that

$$
\|f g\|_{p} \leqq D_{1}(p)\left(\|f\|_{p}\|g\|_{0}+\|f\|_{0}\|g\|_{p}\right), \quad f, g \in D_{p}(\mathscr{T}) .
$$

and $\left(D_{p}(\mathscr{T}), T^{\prime}\right)$ is a differential *-subalgebra of $C(\mathscr{T})$ of order 1 .

Proof. First assume that $\mathscr{T}=[a, b]$ and set $h=b-a$. In [7] it is proved that, for all $k$ and $m, k \leqq m$,

$$
\left\|f^{(k)}\right\| \leqq C(k, m)\|f\|^{(m-k) / m} M_{m}(f)^{k / m}
$$

where $\quad C(k, m)=4 e^{2 k}(m / k)^{k} \quad$ and $\quad M_{m}(f)=\max \left\{\|f\| m ! h^{-m},\left\|f^{(m)}\right\|\right\}$. Let $\quad G(h, m)=$ (m!) $\max \left\{1, m ! h^{-m}\right\}$. Then 


$$
M_{m}(f) \leqq G(h, m)\left(\|f\|+\left\|f^{(m)}\right\| / m !\right) \leqq G(h, m)\|f\|_{m} .
$$

Thus $\left\|f^{(k)}\right\| \leqq R(k, m, h)\|f\|^{(m-k) / m}\|f\|_{m}^{k / m}$ where $R(k, m, h)=C(k, m) G(h, m)^{k / m}$. Hence

$$
\left\|f^{(k)}\right\|\left\|g^{(m-k)}\right\| \leqq R(k, m, h) R(m-k, m, h)\left(\|f\|\|g\|_{m}\right)^{(m-k) / m}\left(\|f\|_{m}\|g\|\right)^{k / m} .
$$

Using the inequality $\alpha^{x} \beta^{1-x} \leqq \alpha x+\beta(1-x), \alpha, \beta \geqq 0,0 \leqq x \leqq 1$, we obtain that

$$
\begin{aligned}
\left\|f^{(k)}\right\|\left\|g^{(m-k)}\right\| & \leqq L(k, m, h)\left(\|f\|\|g\|_{m}(m-k) / m+\|f\|_{m}\|g\| k / m\right) \\
& \leqq L(k, m, h)\left(\|f\|\|g\|_{m}+\|f\|_{m}\|g\|\right)
\end{aligned}
$$

where $L(k, m, h)=,R(k, m, h) R,(m-k, m, h)$. Therefore

$$
\begin{aligned}
\|f g\|_{p} & =\sum_{m=0}^{p}\left\|(f g)^{(m)}\right\| / m ! \leqq \sum_{m=0}^{p} \frac{1}{m !}\left[\sum_{i=0}^{m}\left(\begin{array}{c}
m \\
i
\end{array}\right)\left\|f^{(i)}\right\|\left\|g^{(m-i)}\right\|\right] \\
& \leqq \sum_{m=0}^{p} \frac{1}{m !}\left[\sum_{i=0}^{m}\left(\begin{array}{c}
m \\
i
\end{array}\right) L(i, m, h)\left(\|f\|_{m}\|g\|+\|f\|\|g\|_{m}\right)\right]
\end{aligned}
$$

Since $\|f\|_{m} \leqq\|f\|_{p}$, for $m \leqq p$,

$$
\|f g\|_{p} \leqq D(p, h)\left(\|f\|_{p}\|g\|+\|f\|\|g\|_{p}\right)
$$

where $D(p, h)=\sum_{m=0}^{p} 1 / m !\left[\sum_{i=0}^{m}\left(\begin{array}{c}m \\ i\end{array}\right) L(i, m, h)\right]$.

Assume now that $\mathscr{T}=\bigcup_{i=1}^{n}\left[a_{i}, b_{i}\right], n<\infty$, and let $h_{i}=b_{i}-a_{i}$. For $f \in D_{p}(\mathscr{T})$ and $1 \leqq i \leqq n$, set

$$
\|f\|^{[i]}=\sup _{t \in\left[a_{i}, b_{i}\right]}|f(t)| \text { and }\|f\|_{p}^{[i]}=\sum_{k=0}^{p} \frac{1}{k !}\left\|f^{(k)}\right\|^{[i]}
$$

Then

$$
\|f\|_{0}=\max _{i \leqq i \leqq n}\left(\|f\|_{0}^{[i]}\right) \quad \text { and } \quad \max _{1 \leqq i \leqq n}\left(\|f\|_{p}^{[i]}\right) \leqq\|f\|_{p} \leqq \sum_{i=1}^{n}\|f\|_{p}^{[i]}
$$

Set $D_{1}(p)=\sum_{i=1}^{n} D_{1}\left(p, h_{i}\right)$. Then, by (7), for $f, g \in D_{p}(\mathscr{T})$,

$$
\|f g\|_{p} \leqq \sum_{i=1}^{n}\|f g\|_{p}^{[i]} \leqq D_{1}(p)\left(\|f\|_{p}\|g\|_{0}+\|f\|_{0}\|g\|_{p}\right)
$$


We shall now extend the result of Theorem 8 to generators $\delta$ of strongly continuous one-parameter semigroups of bounded automorphisms $\alpha_{t}$ of Banach algebras $A$. There exists a dense subalgebra $D(\delta)$ in $A$ such that $\delta(x)=\lim _{1 \rightarrow 0}\left(\alpha_{1}(x)-x\right) / t, x \in D(\delta)$, is a closed derivation on $A$. For every $p$,

$$
D\left(\delta^{p}\right)=\left\{x \in D(\delta): \delta^{k}(x) \in D(\delta), 1 \leqq k \leqq p-1\right\}
$$

is a subalgebra of $A$. It is a Banach algebra with respect to the norm $\|x\|_{p}=$ $\sum_{k=0}^{p}\left\|\delta^{(k)}(x)\right\| / k$ ! and $T=\left\{\mid \|_{i}\right\}_{i=0}^{p},|x|_{i}=\left\|\delta^{(i)}(x)\right\| / i$ ! is a flat differential seminorm on $D\left(\delta^{p}\right)$. Since $D\left(\delta^{p}\right)$ is dense in $A$, it is a differential subalgebra of $A$ of order $p$.

Theorem 9. There exists a constant $D_{1}(p)>0$ such that

$$
\|x y\|_{p} \leqq D_{1}(p)\left(\|x\|_{p}\|y\|+\|x\|\|y\|_{p}\right), \quad x, y \in D\left(\delta^{p}\right),
$$

so that $T^{\prime}=\left\{\|\|,\|\|_{p}\right\}$ is a differential seminorm of order 1 on $D\left(\delta^{p}\right)$ equivalent to $T$. Thus $D\left(\delta^{p}\right)$ is a differential subalgebra of $A$ of order 1.

Proof. We have (see [6, v. I]) that, for $t \geqq 0, \alpha_{t}(x) \in D(\delta)$ and $d \alpha_{t}(x) / d t=\delta\left(\alpha_{t}(x)\right)=$ $\alpha_{t}(\delta(x)), x \in D(\delta)$. Therefore if $x \in D\left(\delta^{k}\right)$, then

$$
\alpha_{t}(x) \in D\left(\delta^{k}\right) \quad \text { and } \quad d \alpha_{t}\left(\delta^{k-1}(x)\right) / d t=\delta\left(\alpha_{t}\left(\delta^{k-1}(x)\right)\right)=\alpha_{t}\left(\delta^{k}(x)\right)
$$

Hence $\delta^{k}\left(\alpha_{t}(x)\right)=\alpha_{t}\left(\delta^{k}(x)\right)$.

Let $f$ be a bounded functional on $A$ such that $\|f\|=1$. For $x \in A$, the function $F(x, t)=f\left(\alpha_{t}(x)\right)$ is continuous with respect to $t$. If $x \in D\left(\delta^{p}\right)$, then $F(x, t)$ has $p$ continuous derivatives with respect to $t$ and

$$
F^{(p)}(x, t)=f\left(\delta^{p}\left(\alpha_{t}(x)\right)\right)=f\left(\alpha_{t}\left(\delta^{p}(x)\right)\right)=F\left(\delta^{p}(x), t\right) .
$$

Let $C(\mathscr{T})$ be the $C^{*}$-algebra of all continuous functions on $\mathscr{T}=[0,1]$. If $x \in D\left(\delta^{p}\right)$, $F(x, t) \in D_{p}(\mathscr{T})$. By Lemma VIII.1.3 [6, v. I], there is $C<\infty$ such that $\sup _{0 \leqq} \leqq 1\left\|\alpha_{t}\right\|=C$. Therefore

$$
\|F\|=\sup _{0 \leqq t \leqq 1}\left|f\left(\alpha_{t}(x)\right)\right| \leqq \sup _{0 \leqq t \leqq 1}\left\|\alpha_{t}(x)\right\| \leqq\|x\| \sup _{0 \leqq t \leqq 1}\left\|\alpha_{t}\right\| \leqq C\|x\|
$$

and, for $k \leqq p$,

$$
\left\|F^{(k)}\right\|=\sup _{0 \leqq t \leqq 1}\left|f\left(\alpha_{1}\left(\delta^{k}(x)\right)\right)\right| \leqq \sup _{0 \leqq t \leqq 1}\left\|\alpha_{\imath}\left(\delta^{k}(x)\right)\right\| \leqq C\left\|\delta^{k}(x)\right\| .
$$

Then

$$
\|F\|_{p}=\sum_{k=0}^{p}\left\|F^{(k)}\right\| / k ! \leqq \sum_{k=0}^{p} C\left\|\delta^{(k)}(x)\right\| / k ! \leqq C\|x\|_{p}
$$


Let now $f$ and $g$ be bounded functionals on $A$ such that $\|f\|=\|g\|=1$, let $x, y \in D\left(\delta^{p}\right)$ and let $F(x, t)=f\left(\alpha_{t}(x)\right)$ and $G(y, t)=g\left(\alpha_{t}(y)\right)$. By (6), for every $k \leqq m$, there exists a constant $L(k, m, 1)$ such that

$$
\left\|F^{(k)}\right\|\left\|G^{(m-k)}\right\| \leqq L(k, m, 1)\left(\|F\|_{m}\|G\|+\|F\|\|G\|_{m}\right) .
$$

Therefore

$$
\sup _{0 \leqq 1 \leqq 1}\left|f\left(\alpha_{\imath}\left(\delta^{k}(x)\right)\right)\right| \sup _{0 \leqq t \leqq 1}\left|g\left(\alpha_{\imath}\left(\delta^{m-k}(y)\right)\right)\right| \leqq C L(k, m, 1)\left(\|x\|_{m}\|y\|+\|x\|\|y\|_{m}\right) .
$$

Set $t=0$. Then $\left|f\left(\delta^{k}(x)\right)\right|\left|g\left(\delta^{m-k}(y)\right)\right| \leqq C L(k, m, 1)\left(\|x\|_{m}\|y\|+\|x\|\|y\|_{m}\right)$. Since this inequality holds for all $f, g \in A^{*}$, such that $\|f\|=\|g\|=1$,

$$
\left\|\delta^{k}(x)\right\|\left\|\delta^{m-k}(y)\right\| \leqq C L(k, m, 1)\left(\|x\|_{m}\|y\|+\|x\|\|y\|_{m}\right) .
$$

From this it follows that

$$
\begin{aligned}
\|x y\|_{p} & =\sum_{m=0}^{p}\left\|\delta^{m}(x y)\right\| / m ! \leqq \sum_{m=0}^{p} \frac{1}{m !}\left[\sum_{k=0}^{m}\left(\begin{array}{c}
m \\
k
\end{array}\right)\left\|\delta^{k}(x)\right\|\left\|\delta^{m-k}(y)\right\|\right] \\
& \leqq \sum_{m=0}^{p} \frac{1}{m !}\left[\sum_{k=0}^{m}\left(\begin{array}{c}
m \\
k
\end{array}\right) C L(k, m, 1)\left(\|x\|_{m}\|y\|+\|x\|\|y\|_{m}\right)\right] .
\end{aligned}
$$

Since $\|x\|_{m} \leqq\|x\|_{p}$, for $m \leqq p,\|x y\|_{p} \leqq D_{1}(p)\left(\|x\|_{p}\|y\|+\|x\|\|y\|_{p}\right)$, where

$$
D_{1}(p)=C \sum_{m=0}^{p} \frac{1}{m !} \sum_{k=0}^{m}\left(\begin{array}{l}
m \\
k
\end{array}\right) L(k, m, 1)
$$

Let $\left(A,\|\|_{0}\right)$ be a $C^{*}$-algebra and let $x=x^{*} \in A$. We call $\{a, b\}, a \leqq b$, endpoints in $S p(x)$ if $a, b \in S p(x)$ and there is $\varepsilon>0$ such that

$$
(a-\varepsilon, a) \cap S p(x)=(b, b+\varepsilon) \cap S p(x)=\varnothing .
$$

If $p_{a, b}(t)$ is a continuous function such that $p_{a, b}(t)=1, t \in[a, b]$, and $p_{a, b}(t)=0$, $t \notin(a-\varepsilon, b+\varepsilon)$, then $P_{a, b}(x) \in A$ is a projection and $\left\|p_{a, b}(x)\right\|_{0}=1$.

Corollary 10. Let $\delta$ be a closed *-derivation of a commutative $C^{*}$-algebra $\left(A,\|\|_{0}\right)$ and let $D\left(\delta^{2}\right)$ be a differential subalgebra of order 1 with respect to the norms $\|x\|_{0}$ and $\|x\|_{2}=\|x\|_{0}+\|\delta(x)\|_{0}+\left\|\delta^{2}(x)\right\|_{0} / 2$. Let $y=y^{*} \in D\left(\delta^{2}\right)$ and let there be endpoints $\left\{a_{i}, b_{i}\right\}_{i=0}^{\infty}$ in $S p(y)$ such that $b_{i}-a_{i} \rightarrow 0$, as $i \rightarrow \infty$. Then $\left\|p_{a_{i}, b_{i}}(y) \delta(y)\right\|_{0} \rightarrow 0$. In particular, $\delta(y) \neq 1$.

Proof. Let $g_{i}(t)$ be functions which have two continuous derivatives and such that 
$g_{i}(t)=t-a_{i}, t \in\left[a_{i}, b_{i}\right]$, and $g_{i}(t)=0$, if $t$ is outside $\left(a_{i}-\varepsilon_{i}, b_{i}+\varepsilon_{i}\right)$. Then $g_{i}(y) \in D\left(\delta^{2}\right)$ and $\left\|g_{i}(y)\right\|_{0}=b_{i}-a_{i}$. Since $g_{i}^{\prime}(t)=p_{a_{i}, b_{i}}(t)$ and since $A$ is commutative,

$$
\delta\left(g_{i}(y)\right)=g_{i}^{\prime}(y) \delta(y)=p_{a_{i}, b_{i}}(y) \delta(y) \quad \text { and } \quad p_{a_{i}, b_{i}}(y) \in D(\delta)
$$

For any projection $p$ in $D(\delta), \delta(p)=\delta\left(p^{2}\right)=2 p \delta(p)$, so that $p \delta(p)=2 p \delta(p)$. Therefore $\delta(p)=0$. Hence $\delta\left(p_{a_{i}, b_{i}}(y)\right)=0$ and

$$
\delta^{2}\left(g_{i}(y)\right)=\delta\left(p_{a_{i}, b_{i}}(y) \delta(y)\right)=p_{a_{i}, b_{i}}(y) \delta^{2}(y) .
$$

Thus

$$
\left\|g_{i}(y)\right\|_{2}=\left\|g_{i}(y)\right\|_{0}+\left\|\delta\left(g_{i}(y)\right)\right\|_{0}+\left\|\delta^{2}\left(g_{i}(y)\right)\right\|_{0} / 2 \leqq\left(b_{i}-a_{i}\right)+\|\delta(y)\|_{0}+\left\|\delta^{2}(y)\right\|_{0} / 2 .
$$

Set $h_{i}(y)=\left(g_{i}(y)\right)^{2}$. Then $\left\|h_{i}(y)\right\|_{0}=\left\|g_{i}(y)\right\|_{0}^{2}=\left(b_{i}-a_{i}\right)^{2}$,

$$
\left\|\delta\left(h_{i}(y)\right)\right\|_{0}=\left\|2 g_{i}(y) \delta\left(g_{i}(y)\right)\right\|_{0} \leqq 2\left(b_{i}-a_{i}\right)\|\delta(y)\|_{0}
$$

and

$$
\left\|\delta^{2}\left(h_{i}(y)\right)\right\|_{0}=2\left\|g_{i}(y) \delta^{2}\left(g_{i}(y)\right)+\left[\delta\left(g_{i}(y)\right)\right]^{2}\right\|_{0}=2\left\|g_{i}(y) p_{a_{i}, b_{i}}(y) \delta^{2}(y)+\left[p_{a_{i}, b_{i}}(y) \delta(y)\right]^{2}\right\|_{0} .
$$

Since $D\left(\delta^{2}\right)$ is a differential subalgebra of $A$ of order 1 , there is $D>0$ such that $\left\|\left(g_{i}(y)\right)^{2}\right\|_{2} \leqq 2 D\left\|g_{i}(y)\right\|_{2}\left\|g_{i}(y)\right\|_{0}$. Therefore

$$
\left\|g_{i}(y) p_{a_{i}, b_{i}}(y) \delta^{2}(y)+\left[p_{a_{i}, b_{i}}(y) \delta(y)\right]^{2}\right\|_{0} \leqq 2 D\left[\left(b_{i}-a_{i}\right)+\|\delta(y)\|_{0}+\left\|\delta^{2}(y)\right\|_{0} / 2\right]\left(b_{i}-a_{i}\right) \rightarrow 0 .
$$

as $i \rightarrow \infty$. Hence $\left\|p_{a_{i}, b_{i}}(y) \delta(y)\right\|_{0} \rightarrow 0$.

If we put $n=\infty$ and $\min _{1 \leqq i<\infty}\left(h_{i}\right)=0$ in Theorem 8 , we obtain an example which shows that $T^{\prime}=\left\{\|\|_{0},\|\|_{p}\right\}$ is not necessarily a differential seminorm on $D\left(\delta_{p}\right)$.

Example 5. Let

$$
\mathscr{T}_{1}=\left(\bigcup_{i=1}^{\infty}\left[\frac{2}{2^{i}}, \frac{3}{2^{i}}\right]\right) \bigcup\{0\}
$$

let $A=C\left(\mathscr{T}_{1}\right)$ and let $\delta=(d / d t)$. The function $y=y(t)=t, t \in \mathscr{T}_{1}$, belongs to $D\left(\delta^{2}\right)$ and $\delta(y)=1$. The points $a_{i}=\left(2 / 2^{i}\right)$ and $b_{i}=\left(3 / 2^{i}\right)$ are endpoints of $y$ and $b_{i}-a_{i} \rightarrow 0$ as $i \rightarrow \infty$. By Corollary $10, T^{\prime}=\left\{\|\|_{0},\|\|_{2}\right\}$ is not a differential seminorm on $D\left(\delta^{2}\right)$.

\section{Functional calculus on $\left(D_{p}^{*}\right)$-subalgebras of $C^{*}$-algebras}

Powers [12] (cf. [5] and [10]) proved that if $\delta$ is a closed *-derivation of a $C^{*}$-algebra $A$, if $x=x^{*} \in D(\delta)$ and a function $f(t)$ has two continuous derivatives on $S p_{A}(x)$, then $f(x) \in D(\delta)$. Bratteli, Elliott and Jorgensen [3] generalized this result and showed 
that $f(x) \in D\left(\delta^{p}\right)$ if $f(t)$ has $p+1$ continuous derivatives and $x=x^{*} \in D\left(\delta^{p}\right)$. Blackadar and Cuntz [1] extended this result to differential subalgebras of $C^{*}$-algebras. (D:)-subalgebras are differential subalgebras of order 1, so that Blackadar's and Cuntz's result holds for them. ( $\left.\mathbf{D}_{p}\right)$-subalgebras, $p \geqq 2$, however, are not, generally speaking, differential subalgebras. This section considers some sufficient conditions for $f(x)$ to belong to $B$ if $x^{*}=x \in B$ and $B$ is a $\left(D_{p}^{*}\right)$-subalgebra of $A$. This will allows us to show that $\left(\mathbf{D}_{p}^{*}\right)$-subalgebras of $C^{*}$-algebras are locally normal.

Lemma 11. If $B$ is a ( $\left.\mathrm{D}_{1}^{*}\right)$-subalgebra of a unital $C^{*}$-algebra $A$, then $\|\mathbf{1}\|_{1}=1$ and $D_{1}>1 / 2$.

Proof. By Theorem $5,1 \in B$. Therefore $\|1\|_{1}=\left\|1^{2}\right\|_{1} \leqq 2 D_{1}\|1\|_{1}\|1\|_{0}$, so that $1 / 2 \leqq D_{1}$. Let $D_{1}=1 / 2$. By Lemma 1 , for $x^{*}=x \in B$ and for all $k$,

$$
\begin{aligned}
\|\exp (i x)\|_{1} & =\|\left(\operatorname { e x p } ( i x / 2 ^ { k } ) ^ { 2 ^ { k } } \| _ { 1 } \leqq ( 2 D _ { 1 } ) ^ { k } \| \operatorname { e x p } ( i x / 2 ^ { k } ) \| _ { 0 } ^ { 2 ^ { k } - 1 } \| \left(\exp \left(i x / 2^{k}\right) \|_{1}\right.\right. \\
& =\|\left(\exp \left(i x / 2^{k}\right) \|_{1} \leqq \exp \left(\|x\|_{1} / 2^{k}\right) \rightarrow 1\right.
\end{aligned}
$$

as $k \rightarrow \infty$, since $\left\|\exp \left(i x / 2^{k}\right)\right\|_{0}=1$. Therefore

$$
\|\mathbf{1}\|_{1}=\|\exp (i x) \exp (-i x)\|_{1} \leqq\|\exp (i x)\|_{1}\|\exp (-i x)\|_{1} \leqq 1,
$$

so that $\|1\|_{1}=1$ and $\|\exp (i x)\|_{1}=1$. Since $\left(B,\|\|_{1}\right)$ is a Banach *-algebra, it follows from Theorem 38.14 [2] that $B$ is a $C^{*}$-algebra. Since $B$ is dense in $A, B=A$. Thus $D_{1}>1 / 2$.

Theorem 12. Let $B$ be a dense *-subalgebra of a $C^{*}$-algebra $\left(A,\|\|_{0}\right)$ with identity, let $x=x^{*} \in B$ and let $[a, b]$ contain $S p_{A}(x)$. Let $f(t)$ be a continuous function on $(-\infty, \infty)$ such that $f(t)=0$ outside $[a, b]$ and let $\hat{f}(s)$ be its Fourier transform.

(i) Let $B$ be a ( $\left.\mathrm{D}_{p}^{*}\right)$-subalgebra of $A$ and $M=\max _{1 \leqq j \leqq p}\left\{2 D_{j}\right.$, $\left.\exp \left(\|x\|_{j}\right)\right\}$. If $\int_{-\infty}^{\infty}|2 s|^{\left(\lg _{2}|2 s|\right)^{p-1} \lg _{2} M /(p-1) !}|f(s)| d s<\infty$, for $p \geqq 2$, or $\int_{-\infty}^{\infty}|2 s|^{1+\lg _{2}\left(D_{1}\right)}|\hat{f}(s)| d s<\infty$, for $p=1$, then $f(x) \in B$.

(ii) Let $B$ be a ( $\left.\mathbf{D}_{1}^{*}\right)$-subalgebra of $A$. If $f(t)$ has $q \geqq 2+\lg _{2}\left(D_{1}\right)$ continuous derivatives, then $f(x) \in B$.

(iii) (cf. [1)]. Let $B$ be a differential subalgebra of $A$ with respect to a differential seminorm $T=\left\{\|_{i}\right\}_{i=0}^{p}$, where $\left\|_{0}=\right\| \|_{0}$, and let $N$ be the total order of $T$ (see Lemma 7). If $\int_{-\infty}^{\infty}|2 s|^{l g_{2}(N)}|\hat{f}(s)| d s<\infty$ or if $f(t)$ has $q \geqq 1+\lg _{2}(N)$ continuous derivatives on $[a, b]$, then $f(x) \in B$.

Proof. By Theorem $5,1 \in B$, so that $\exp (i s x) \in B$ for real $s$. Let $k$ be the integer such that $2^{k-1}<|s| \leqq 2^{k}$, so that $k-1<\lg _{2}|s| \leqq k$. Set $y=i s x / 2^{k}$. Then $\|\exp (y)\|_{0}=1$ and

$$
\|\exp (y)\|_{j}=\left\|\exp \left(i s x / 2^{k}\right)\right\|_{j} \leqq \exp \left(|s|\|x\|_{j} / 2^{k} \mid \leqq M .\right.
$$


for $1 \leqq j \leqq p$. For every $z \in B,\left\|z^{2}\right\|_{i} \leqq 2 D_{i}\|z\|_{i}\|z\|_{i-1}$. Making use of Lemma 1 and replacing there $C_{i}$ by $2 D_{i}$, we obtain

$$
\begin{aligned}
\|\exp (i s x)\|_{p} & =\left\|\left(\exp \left(2^{k} y\right)\right)\right\|_{p}=\left\|(\exp (y))^{2^{k}}\right\|_{p} \\
& \leqq\|\exp (y)\|_{0}^{2^{k}-S(k, p)} \prod_{j=0}^{p-1}\|\exp (y)\|_{p-j}^{a(k, j)}\left(2 D_{p-j}\right)^{a(k, j+1)} \\
& =\prod_{j=0}^{p-1}\|\exp (y)\|_{p-j}^{a(k, j)}\left(2 D_{p-j}\right)^{a(k, j+1)} \leqq M^{b}
\end{aligned}
$$

where $a(k, j)=\left(\begin{array}{l}k \\ j\end{array}\right)$ and $b=\sum_{j=0}^{p-1}[a(k, j)+a(k, j+1)]$. For $2 p<k$

$$
b \leqq 1+2(p-1) a(k, p-1)+a(k, p) \leqq 1+2(p-1) k^{p-1} /(p-1) !+k^{p} / p ! \leqq 1+k^{p} /(p-1) !
$$

Since $1 \leqq M$,

$$
\|\exp (i s x)\|_{p} \leqq M^{1+k^{p} /(p-1) !} \leqq M^{1+(l g 2|s|+1)^{p} /(p-1) !}=M|2 s|^{\psi(s)}
$$

where $\psi(s)=\left(\lg _{2}|2 s|\right)^{p-1} \lg _{2} M /(p-1) !$.

The rest of the proof of (i) follows the proof of Proposition 3.3.6 [14] with insignificant changes. Since $\hat{f}$ is continuous on $(-\infty, \infty)$ and vanishes at infinity and since $\lg _{2} M \geqq 0, \int_{-\infty}^{\infty}|2 s|^{\psi(s)}|\hat{f}(s)| d s<\infty$ implies $\int_{-\infty}^{\infty}|\hat{f}(s)| d s<\infty$. Since $f$ is continuous and

$$
\hat{f}(t)=1 /(2 \pi)^{1 / 2} \int_{-\infty}^{\infty} \exp (i t s) \hat{f}(s) d s
$$

is continuous and since $\hat{\jmath}=f$ almost everywhere, $\hat{f}=f$. Therefore

$$
f(x(\lambda))=1 /(2 \pi)^{1 / 2} \int_{-\infty}^{\infty} \exp (i x(\lambda) s) \hat{f}(s) d s, \text { for } \lambda \in S p_{A}(x)
$$

It follows from (9) that

$$
\int_{-\infty}^{\infty}\|\exp (i x s)\|_{p}|\hat{f}(s)| d s \leqq \int_{-\infty}^{\infty} M|2 s|^{\psi(s)}|\hat{f}(s)| d s<\infty
$$

Therefore $\int_{-\infty}^{\infty} \exp (i x s) f(s) d s$ is absolutely convergent in \|\|$_{p}$. Hence

$$
y=1 /(2 \pi)^{1 / 2} \int_{-\infty}^{\infty} \exp (i x s) \hat{f}(s) d s \in B \quad \text { and } \quad y(\lambda)=f(x(\lambda))
$$


for $\lambda \in S p_{A}(x)$. Thus $y=f(x) \in B$.

For $p=1$, it follows from (8) that

$$
\|\exp (i s x)\|_{1} \leqq\|\exp (y)\|_{1}\left(2 D_{1}\right)^{k} \leqq M\left(2 D_{1}\right)^{l g_{2}|2 s|}=M|2 s|^{1+\lg _{2}\left(D_{1}\right)} .
$$

Repeating the above argument, we obtain that $f(x) \in B$ if $\int_{-\infty}^{\infty}|s|^{1+\lg _{2}\left(D_{1}\right)}|\hat{f}(s)| d s<\infty$. Part (i) is proved.

Part (ii) follows from (i) and from the proof of Theorem 3.3.7 [14].

It follows from Lemma 7 that

$$
\|\exp (i s x)\|_{p}=\left\|(\exp (i t x))^{2^{k}}\right\|_{p} \leqq K\left((\exp (i t x)) N^{k}\right.
$$

where $t=s / 2^{k}$, so that $1 / 2 \leqq|t| \leqq 1$. Since $K(\exp (i t x))$ is continuous with respect to \|\|$_{p}$, there exists $M(x)=\sup _{1 / 2 \leqq 1 \leqq 1} K(\exp (i t x))<\infty$. Hence

$$
\|\exp (i s x)\|_{p} \leqq M(x) N^{\lg _{2}(2|s|)}=M(x)|2 s|^{\lg (N)}
$$

and this case is similar to the case of $\left(D_{1}^{*}\right)$-subalgebra of $A$ where $1+\lg _{2}\left(D_{1}\right)$ is substituted by $\lg _{2}(N)$.

Recall that a family $F$ of functions on a topological space $X$ is said to be normal (see, for example, $[11, \S 15])$ if for any disjoint closed subsets $S$ and $T$ in $X$, there exists a function $f \in F$ such that

$$
f(x)=0 \text { on } T \text { and } f(x)=1 \text { on } S .
$$

Definition. Let $B$ be a dense subalgebra of a Banach algebra $A$ with an identity 1 and let $1 \in B$.

(1) Let $A$ be commutative. The algebra $B$ is said to be normal if the algebra of functions $\{x(s): x \in B\}$ on the space of all maximal ideals of $A$ is normal.

(2) Let $A$ and $B$ be *-algebras. Then $B$ is said to be locally normal if for every selfadjoint $x \in B$, there is a commutative Banach *-subalgebra $A(x)$ in $A$ such that 1 and $x$ belong to $A(x)$ and such that $B(x)=B \cap A(x)$ is a dense normal subalgebra of $A(x)$.

Theorem 13. Let $\left(A,\|\|_{0}\right)$ be a $C^{*}$-algebra with identity and let $B$ be a $\left(\mathbf{D}_{p}^{*}\right)$-subalgebra of $A$. Then

(i) $B$ is a locally normal $Q^{*}$-subalgebra of $A$;

(ii) everywhere defined derivations from $B$ into $A$ are bounded;

(iii) the mapping $I \rightarrow I \cap B$ is a one-to-one mapping of the set of all closed two-sided ideals in $A$ onto the set of all closed (in the topology of $A$ ) two-sided ideals in $B$ and of the set of all maximal ideals of $A$ onto the set of all maximal ideals of $B$. 
Proof. By Theorem 5, $1 \in B$. It was shown in (9) that, for $x=x^{*} \in B, \| \exp ($ is $x) \|_{p} \leqq$ $M|2 s|^{\psi(s)}$, where $\psi(s)=\left(\lg _{2}|2 s|\right)^{p-1} \lg _{2} M /(p-1) ! \quad$ and $M=\max _{1 \leqq j \leqq p}\left\{2 D_{j}, \exp \left(\|x\|_{j}\right)\right\}$.

Therefore

$$
\int_{-\infty}^{\infty} \ln \|\exp (i s x)\|_{p} \frac{d s}{1+s^{2}}<\infty
$$

It follows from the Shilov's condition of regularity $([11, \S 15,6])$ that $B$ is locally normal. Part (ii) follows from (i) and from [9] and part (iii) follows from (i) and from [8].

\section{Banach *-algebras isomorphic to $\left(D_{1}^{*}\right)$-subalgebras of $C^{*}$-algebra}

$\left(D_{1}^{*}\right)$-subalgebras of $C^{*}$-algebras constitute the simplest and the most interesting subclass of $C^{*}$-algebras. In Section 3 we showed that even some differential subalgebras of order $p \geqq 2$ are, in fact, $\left(\mathbf{D}_{1}^{*}\right)$-subalgebras. In this section we characterize those Banach *-algebras which are isomorphic to $\left(\mathbf{D}_{1}^{*}\right)$-subalgebras of $C^{*}$-algebras.

Definition. Let $(B,\|\|)$ be a *-Banach algebra and $r_{B}$ be the spectral radius on $B$. We say that $B$ has property $\left(D^{*}, r\right)$ if there exists $D \geqq 0$ such that

$$
\|x y\| \leqq D\left(\|x\| r_{B}(y)+\|y\| r_{B}(x)\right), \text { for } x=x^{*} \in B \text { and } y=y^{*} \in B \text {. }
$$

Example 6. Let $\mathscr{B}$ be a $C^{*}$-algebra with a norm $\|$.

(1) Let

$$
B=\left\{b=\left(\begin{array}{cc}
x & y \\
0 & x
\end{array}\right): x, y \in \mathscr{B}\right\}, \quad b^{*}=\left(\begin{array}{cc}
x^{*} & y^{*} \\
0 & x^{*}
\end{array}\right) \quad \text { and }\|b\|=|x|+|y| .
$$

Then $B$ is a Banach *-algebra and the radical

$$
R(B)=\left\{\left(\begin{array}{ll}
0 & y \\
0 & 0
\end{array}\right): y \in \mathscr{B}\right\}
$$

We have that $S p_{\mathscr{P}}(x)=S p_{B}(b)$, so that $r_{\mathscr{P}}(x)=r_{B}(b)$. If $b=b^{*}$, then $x=x^{*}$, so that $r_{B}(b)=|x|$. If $b_{1}^{*}=b_{1} \in B$ and $b_{2}^{*}=b_{2} \in B$, then

$$
\begin{aligned}
& \left\|b_{1} b_{2}\right\|=\left|x_{1} x_{2}\right|+\left|x_{1} y_{2}+y_{1} x_{2}\right| \leqq\left|x_{1}\right| r_{B}\left(b_{2}\right) \\
& \quad+\left|y_{2}\right| r_{B}\left(b_{1}\right)+\left|y_{1}\right| r_{B}\left(b_{2}\right) \leqq\left\|b_{1}\right\| r_{B}\left(b_{2}\right)+\left\|b_{2}\right\| r_{B}\left(b_{1}\right) .
\end{aligned}
$$


Hence $B$ has property $\left(D^{*}, r\right)$.

(2) Let $\delta$ be a closed *-derivation of $\mathscr{B}$. Then $D(\delta)$ is a $\left(\mathbf{D}_{1}^{*}\right)$-subalgebra of $\mathscr{B}$ with respect to the norms $|x|$ and $\|x\|=|x|+|\delta(x)|$. Therefore

$$
\|x y\| \leqq\|x\||y|+|x|\|y\|, \quad x, y \in B,
$$

and, by Theorem $5, D(\delta)$ is a $Q$-subalgebra of $\mathscr{B}$. Hence, by Lemma 4 , for $x=x^{*} \in D(\delta)$, $r_{B}(x)=r_{\mathscr{B}}(x)=|x|$, so that

$$
\|x y\| \leqq\|x\| r_{B}(y)+\|y\| r_{B}(x), \quad x=x^{*} \in B \quad \text { and } \quad y=y^{*} \in B .
$$

Therefore $B$ has property $\left(D^{*}, r\right)$.

Let $B$ be a Banach *-algebra and let $\mathscr{P}(B)$ be the set of all positive functionals on $B$. Then (see $[11, \S 18,2])$

$$
I(B)=\left\{x \in B: f\left(x^{*} x\right)=0 \text { for all } f \in \mathscr{P}(B)\right\}
$$

is a closed symmetric two-sided ideal of $B$ and the radical $R(B) \subseteq I(B)$. A Banach *-algebra is called reduced if $I(B)=\{0\}$.

Theorem 14. Let $(B,\|\|)$ be a Banach *-algebra with identity. The following conditions are equivalent:

(i) $B$ is a reduced algebra with property $\left(\mathbf{D}_{1}^{*}, r\right)$;

(ii) $B$ is a $\left(\mathbf{D}_{1}^{*}\right)$-subalgebra of a $C^{*}$-algebra;

(iii) $B$ is a reduced algebra and there exists $D>0$ such that $\left\|x^{*} x\right\| \leqq 2 D\|x\| r_{B}\left(x^{*} x\right)^{1 / 2}$, for all $x \in B$.

Proof. (ii) $\Rightarrow$ (i). Let $\left(A,\|\|_{0}\right)$ be a $C^{*}$-algebra with identity, let $B$ be a dense *-subalgebra of $A$ and let there exist $D_{1}>0$ such that $\|x y\| \leqq D_{1}\left(\|x\|\|y\|_{0}+\|x\|_{0}\|y\|\right)$, $x, y \in B$. If $r_{A}$ is the spectral radius on $A$, then, for selfadjoint $x,\|x\|_{0}=r_{A}(x)$, so that

$$
\|x y\| \leqq D_{1}\left(\|x\| r_{A}(y)+\|y\| r_{A}(x)\right), \text { for } x=x^{*} \in B \text { and } y=y^{*} \in B
$$

By Theorem $5, B$ is a $Q^{*}$-subalgebra of $A$. Therefore it follows from Lemma 4 that $r_{A}(x)=r_{B}(x)$ and we obtain that

$$
\|x y\| \leqq D_{1}\left(\|x\| r_{B}(y)+\|y\| r_{B}(x)\right), \text { for } x=x^{*} \in B \text { and } y=y^{*} \in B
$$

Thus $B$ has property $\left(D^{*}, r\right)$.

Since $A$ is reduced, $B$ is also reduced, so that $B$ is a reduced algebra with property $\left(D^{*}, r\right)$.

(ii) $\Rightarrow$ (iii). Since $B$ is a $\left(D_{i}^{*}\right)$-subalgebra of $A$, 


$$
\left\|x^{*} x\right\| \leqq D_{1}\left(\left\|x^{*}\right\|\|x\|_{0}+\left\|x^{*}\right\|_{0}\|x\|\right)=2 D_{1}\|x\|\|x\|_{0}
$$

Since $A$ is a $C^{*}$-algebra, $\|x\|_{0}^{2}=\left\|x^{*} x\right\|_{0}=r_{A}\left(x^{*} x\right)$. Since $B$ is a $Q^{*}$-subalgebra of $A$, it follows from Lemma 4 that $r_{B}\left(x^{*} x\right)=r_{A}\left(x^{*} x\right)$. Hence $\left\|x^{*} x\right\| \leqq 2 D_{1}\|x\| r_{B}\left(x^{*} x\right)^{1 / 2}$. Since $A$ is reduced, $B$ is also reduced.

(i) $\Rightarrow$ (ii). Let $B$ be a reduced algebra with property $\left(D^{*}, r\right)$. Then

$$
\|x\|_{0}=\left(\sup _{f \in \mathscr{P}(B)} f\left(x^{*} x\right)\right)^{1 / 2}, \quad x \in B
$$

is a norm on $B$ such that the completion $A$ of $B$ with respect to \|\|$_{0}$ is the enveloping $C^{*}$-algebra (see $[11, \S 18,3]$ ).

Let $x=x^{*} \in B$ and let $M$ be a maximal commutative *-subalgebra of $B$ which contains $x$. Then $S p_{B}(z)=S p_{M}(z)$ for all $z \in M$. If $z=z^{*}$ belongs to the radical of $M, r_{B}(z)=r_{M}(z)=$ 0 and $\left\|z^{2}\right\| \leqq 2 D_{1}\|z\| r_{B}(z)=0$. Hence $z^{2}=0$ and $z \in I(B)$. Since, by assumption, $I(B)=\{0\}$, $M$ is semisimple.

Let $T$ be the space of all maximal ideals of the algebra $M$ and let $C(T)$ be the $C^{*}$ algebra of all continuous functions on $T$ with the norm $|f|=\sup _{\mathrm{s} \in T}|f(t)|, f \in C(T)$. Then $M$ is a dense *-subalgebra of $C(T)$ and

$$
r_{B}(z)=r_{M}(z)=\sup _{t \in T}|z(t)|=|z|, \text { for all } z \in M .
$$

Therefore, for $z=z^{*} \in M$ and $u=u^{*} \in M$,

$$
\|z u\| \leqq 2 D\left(\|z\| r_{B}(u)+\|u\| r_{B}(z)\right)=2 D(\|z\||u|+|z| \| u \mid)
$$

and, by Theorem $13, M$ is a locally normal $Q^{*}$-subalgebra of $C(T)$.

In Theorem 8 [8] it was proved that if $F$ is a dense locally normal $Q^{*}$-subalgebra of a $C^{*}$-algebra $\mathscr{F}$ and $\pi$ is an injective ${ }^{*}$-homomorphism of $F$ into a $C^{*}$-algebra $\mathscr{A}$, then $\|\pi(y)\|_{\mathscr{A}}=\|y\|_{\mathscr{F}}$ for all $y \in F$. Hence, since $M$ is injectively imbedded in $A$, we obtain that $\|z\|_{0}=|z|$, for all $z \in M$. Therefore $r_{B}(x)=|x|=\|x\|_{0}$. From this it follows that for $x=x^{*} \in B$ and $y=y^{*} \in B$,

$$
\|x y\| \leqq D\left(\|x\| r_{B}(y)+\|y\| r_{B}(x)\right)=D\left(\|x\|\|y\|_{0}+\|x\|_{0}\|y\|\right) .
$$

Now let $z=x+i y \in B$. Then $x=\left(z+z^{*}\right) / 2$ and $y=\left(z-z^{*}\right) / 2 i$ are selfadjoint, $\|x\|_{j} \leqq\|z\|_{j}$ and $\|y\|_{j} \leqq\|z\|_{j}, j=0,1$. Hence, by (10),

$$
\begin{gathered}
\left\|z^{*} z\right\|=\left\|x^{2}+y^{2}+i(x y-y x)\right\| \leqq\left\|x^{2}\right\|+\left\|y^{2}\right\|+\|x y\|+\|y x\| \leqq 2 D\|x\|\|x\|_{0}+2 D\|y\|\|y\|_{0} \\
+2 D\left(\|x\|\|y\|_{0}+\|x\|_{0}\|y\|\right) \leqq 8 D\|z\|\|z\|_{0} .
\end{gathered}
$$

It follows from Lemma 3 that $B$ has property $\left(D_{1}\right)$. 
(iii) $\Rightarrow$ (ii). Let, as above, $A$ be the enveloping $C^{*}$-algebra of $B$, let $x=x^{*} \in B$ and $M$ be a maximal commutative *-subalgebra of $B$ which contains $x$. Then, as in (i) $\Rightarrow$ (ii), we obtain that $M$ is semisimple and that $r_{B}(z)=r_{M}(z)=|z|$ for all $z \in M$. Therefore

$$
\left\|z^{*} z\right\| \leqq 2 D\|z\| r_{B}\left(z^{*} z\right)^{1 / 2}=2 D\|z\|\left|z^{*} z\right|^{1 / 2}=2 D\|z\||z| \text {. }
$$

From Lemma 3 it follows that $M$ is a $\left(\mathbf{D}_{1}^{*}\right)$-subalgebra of $C(T)$. Hence, by Theorem $13, M$ is a locally normal $Q^{*}$-subalgebra of $C(T)$. Then, as in (i) $\Rightarrow(\mathrm{ii}),\|z\|_{0}=|z|$. for $z \in M$. Therefore $r_{B}(x)=|x|=\|x\|_{0}$. Therefore, for every $y \in B$,

$$
\left\|y^{*} y\right\| \leqq 2 D\|y\| r_{B}\left(y^{*} y\right)^{1 / 2}=2 D\|y\|\left\|y^{*} y\right\|_{0}^{1 / 2}=2 D\|y\|\|y\|_{0} .
$$

It follows from Lemma 3 that $B$ is a $\left(D_{1}^{*}\right)$-subalgebra of $A$.

Recall that a Banach *-algebra $B$ is called symmetric if $1+x^{*} x$ is invertible for all $x \in B$. For a symmetric algebra $B, R(B)=I(B)$ (see $[11, \S 23,3]$ ). The following lemma shows that the condition $R(B)=I(B)$ is sufficient for an algebra with property $\left(D^{*}, r\right)$ to be symmetric.

Lemma 15. Let $B$ be a Banach*-algebra with identity and let $B$ have property $\left(D^{*}, r\right)$.

(i) For every $z \in R(B), z^{2}=0$ and the semisimple Banach *-algebra $B / R(B)$ also has property $\left(\mathrm{D}^{*}, r\right)$.

(ii) If $R(B)=I(B)$, then the algebra $B$ is symmetric.

Proof. If $z=z^{*} \in R(B)$, then $\left\|z^{2}\right\| \leqq 2 D\|z\| r_{B}(z)=0$, so that $z^{2}=0$. If $z=y+i u \in R(B)$, $y=y^{*} \in R(B)$ and $u=u^{*} \in R(B)$, then $y^{2}=u^{2}=0$ and $z^{2}=y^{2}+i(y u+u y)-u^{2}=$ $i\left[(y+u)^{2}-y^{2}-u^{2}\right]=0$.

Let $x \rightarrow \hat{x}$ be the canonical mapping of $B$ onto the quotient Banach *algebra $\hat{B}=$ $B / R(B)$. If $z \in R(B)$, then $1+z$ is invertible. If $x \in B$ is invertible, then $x+z=x\left(1+x^{-1} z\right)$ is also invertible. Therefore $S p_{B}(x)=S p_{B}(x+z)$ and $r_{B}(x)=r_{B}(x+z)$. From this it follows that

$$
S p_{B}(x)=S p_{\hat{B}}(\hat{x}) \quad \text { and } \quad r_{B}(x)=r_{\hat{B}}(\hat{x})
$$

Hence, for selfadjoint $x$ and $y$,

$$
\begin{aligned}
\|\hat{x} \hat{y}\| & =\inf _{z \in R(B)}\|x y+z\| \leqq \inf _{z \in R(B)}\|(x+z)(y+z)\| \leqq \\
& \leqq D \inf _{z=z^{*} \in R(B)}\left(\|x+z\| r_{B}(y+z)+\|y+z\| r_{B}(x+z)\right) \\
& \leqq D\left(r_{B}(\hat{y}) \inf _{z=z^{*} \in R(B)}\|x+z\|+r_{B}(\hat{x}) \inf _{z=z^{*} \in R(B)}\|y+z\|\right) .
\end{aligned}
$$

For all selfadjoint $u$ and $z, x+z=[(x+z+i u)+(x+z-i u)] / 2$, so that

$$
\|x+z\| \leqq(\|x+z+i u\|+\|x+z-i u\|) / 2=\|x+z+i u\| .
$$

Therefore $\inf _{z=z^{*} \in R(B)}\|x+z\|=\inf _{z \in R(B)}\|x+z\|=\|\hat{x}\|$. Hence 


$$
\|\hat{x} \hat{y}\| \leqq D\left(\|\hat{x}\| r_{\bar{B}}(\hat{y})+\|\hat{y}\| r_{\hat{B}}(\hat{x})\right), \quad \hat{x}^{*}=\hat{x} \in \hat{B} \quad \text { and } \quad \hat{y}^{*}=\hat{y} \in \hat{B},
$$

and $\hat{B}$ has property $\left(D^{*}, r\right)$. Part (i) is proved.

If $I(B)=R(B)$, the algebra $\hat{B}$ is reduced and has property $\left(D^{*}, r\right)$. Let $A$ be the enveloping $C^{*}$-algebra of $\hat{B}$. By (i) and by Theorem $14, \hat{B}$ is a $\left(\mathbf{D}_{i}^{*}\right)$-subalgebra of $A$. It then follows from Theorem 5 that $\hat{B}$ is a $Q^{*}$-subalgebra of $A$. From this and from (11) we obtain that

$$
S p_{B}\left(1+x^{*} x\right)=S p_{\bar{B}}\left(\hat{\mathbf{1}}+\hat{x}^{*} \hat{x}\right)=S p_{A}\left(\hat{\mathbf{1}}+\hat{\mathbf{x}}^{*} \hat{x}\right) \text { for all } x \in B .
$$

Since $\hat{\mathbf{1}}+\hat{x}^{*} \hat{x}$ is invertible in $A, \mathbf{1}+x^{*} x$ is invertible in $B$. Thus $B$ is a symmetric algebra.

\section{REFERENCES}

1. B. Blackadar and J. Cuntz, Differential Banach algebra norms and smooth subalgebras of $C^{*}$-algebras, J. Operator Theory 26 (1991), 255-282.

2. F. F. Bonsal and J. DunCan, Complete normed algebras (Springer-Verlag, 1973).

3. O. Bratteli, G. A. Elliott and P. E. T. Jorgensen, Decomposition of unbounded derivations into invariant and approximately inner parts, J. Reine Angew. Math. 346 (1984), 166-193.

4. O. Bratteli and D. W. Robinson, Unbounded derivations of $C^{*}$-algebras, I\& II Comm. Math. Phys. 42 (1975), 255-268 and 46 (1976), 11-30.

5. O. Bratteli and D. W. Robinson, Operator algebras and Quantum Statistical Mechanics (Springer-Verlag, 1979).

6. N. Dunford and J. T. Schwartz, Linear Operators, I\& II (Interscience Publ., New York, London, 1963).

7. G. H. Hardy, J. E. Littlewood and G. Polya, Inequalities (Cambridge Unviersity Press, 1934).

8. E. Kissin and V. S. Shulman, Dense $Q$-subalgebras of Banach and $C^{*}$-algebras and unbounded derivations of Banach and $C^{*}$-algebras, Proc. Edinburgh Math. Soc., 36 (1993), 261-276.

9. R. Longo, Automatic relative boundedness of derivations in $C^{*}$-algebras, J. Funct. Anal. 34 (1979), 21-28.

10. A. McIıntosh, Functions and derivations of $C^{*}$-algebras, J. Funct. Anal. 30 (1978), 264-275. 1972).

11. M. A. Naimark, Normed algebras (Wolters-Noordhoff Publishing, Groningen, Netherlands,

12. R. T. Powers, A remark on the domain of an unbounded derivation of a $C^{*}$-algebra, $J$. Funct. Anal. 18 (1975), 85-95.

13. H. ReIter, $L^{1}$-algebras and Segal algebras (Lecture Notes in Mathematics 231, SpringerVerlag, 1971).

14. S. SAKal, Operator algebras in dynamical systems (Cambridge University Press, Cambridge, 1991).

School of Mathematical Sciences

UNIVERSITY OF NORTH LONDON

HOLLOWAY

LONDON N7 8DB

ENGLAND
Department of Mathematics

Polytechnic University of Vologda Vologda

Russia 